\title{
Desarrollo Rural Endógeno: Condiciones para una Transición Agroecológica desde una Experiencia de Producción Orgánica.
}

\author{
Santiago Felipe Peredo y Claudia Paz Barrera ${ }^{1}$
}

\begin{abstract}
The current situation of the peasent agriculture in Chile is characteristed by an increasing socioeconomical margination and degradation of the natural resources. One alternative of rural development to approach upon this problem is the Agroecology that could design development strategy by sustainable agrarian production systems.
\end{abstract}

The aim of this work is to establish those conditions required for the agroecological transition on the basis an organic farming case. In the present article are discussed these conditions.
Ante la actual situación de marginalidad y desarticulación social en que se encuentra la agricultura familiar campesina en un mundo rural caracterizado por condiciones de pobreza tanto económica como ecológica, la Agroecología surge como una alternativa de desarrollo rural basada en estrategias que potencien tanto la diversidad ecológica como sociocultural de una comunidad.

En este trabajo se discuten, a partir de un estudio de caso, las condiciones que se deben reunir en un proceso de transición agroecológica a partir del establecimiento de unidades productivas de naturaleza ecológica (orgánica) en explotaciones campesinas.

\footnotetext{
1 Instituto de Sociología y Estudios Campesinos, Universidad de Córdoba, España. E mail: ec1segue@uco.es
} 


\section{Introducción}

La agricultura familiar campesina en Chile, representada por alrededor de unas 278 mil familias (Odepa, 2001), se encuentra en una situación de marginación creciente y de desarticulación social y económica (Chonchol, 1994; Díaz, 1995), que ha generado condiciones de pobreza en el sector rural caracterizada por el deterioro constante de los recursos naturales, la dificultad para acceder a beneficios productivos y sociales, y una gran informalidad en la tenencia de las tierras y aguas, dificultando con ello, el acceso a créditos y a la asistencia técnica.

Estas tendencias desarticuladoras de la sociedad rural, en donde el acceso a los mercados es desigual, los créditos son restrictivos, las tecnologías son cultural y económicamente inadecuadas y, la ausencia de una política de fomento a las organizaciones sociales y de una institucionalidad adecuada para este sector, ha generado, a juicio de Díaz (op.cit.), una condición de "inviabilidad" dentro de la agricultura campesina.

Bajo esta situación, surge la necesidad de definir esquemas de desarrollo basados en el potencial endógeno -cultural y ecológico- (Peredo y Barrera, 2001a) de la población, a través de formas de producción no degradantes de la naturaleza ni de la sociedad, para abandonar propuestas, que hasta la fecha, solo han propiciado la reproducción de sistemas altamente dependientes y demandantes de energía y materiales exógenos (Sevilla y González de Molina, 1993).

La Agroecología como una alternativa de desarrollo rural (Guzmán et al, 2001), que surge como una respuesta para encarar la crisis ecológica y el problema social generado por ésta. Sobre la base del manejo sostenible de los recursos naturales y el acceso igualitario a los mismos (Altieri, 1987; Sevilla, 1997), se han desarrollado numerosas experiencias (Altieri, 1995; Sevilla, 2001a) donde se ha demostrado que es posible el establecimiento de sistemas de producción sustentables, debido a las ventajas y beneficios que una propuesta agroecológica presenta tanto en sus dimensiones sociales (Sevilla, 2001), culturales (Toledo, 2001), económicas (Martínez, 1994), agronómicas (Altieri, 1999a) y ecológicas (Gliessmann, 1998).

El marco teórico, de carácter universal (y no por ello unívoco o normativo) sobre el cual descansa una propuesta de este tipo (Altieri, 1987; Sevilla, 1997; Altieri y Nicholls, 1999; Guzmán et al, op.cit.), permite definir estrategias de desarrollo bajo contextos particulares y específicos y desde la propia identidad del agroecosistema como una manera de acompañar procesos que están en marcha (Peredo y Sevilla, 2000).

Esta estrategia, de carácter participativa, requiere el establecimiento de ciertos elementos dinamizadores orientados a conducir un proceso de transición, de naturaleza agroecológica, para conseguir un estado superior de "modernidad alternativa" (Toledo, 2000) sobre la base de la praxis del modo campesino (Guha y Gadgil, 1993) o secundario (Toledo, 1994) de uso o apropiación de los recursos naturales.

El presente trabajo corresponde a lo que hemos denominado etapa I de una investigación más amplia (Peredo, 2001) y tiene por objetivo definir aquellos elementos dinámicos que permiten llevar a cabo una transición agroecológica a partir del establecimiento de unidades productivas de carácter orgánico (ecológico). 


\section{Metodología}

Como lo señaláramos en el apartado anterior, este trabajo da cuenta de manera breve y resumida de la etapa I de una investigación más amplia desarrollada en conjunto con grupos de campesinos de la comuna de Chépica de la VI Región.

El tipo de investigación es lo que se conoce como investigación-acción-participativa (IAP), en donde en esta etapa I se llevaron a cabo la $1^{\circ}$ Fase, correspondiente a la fase de acercamiento y conocimiento de la realidad estudiada, y parte de la $2^{\circ}$ Fase, de la investigación participativa (Guzmán et al, 1996), en la que se definieron las unidades productivas en cada grupo sobre la base de los intereses y requerimientos de los propios grupos campesinos. Los grupos estudiados fueron, el Maitén, localidad ubicada a $12 \mathrm{~km}$. de la ciudad de Chépica y el lugar donde se realizó la experiencia productiva se encuentra en una zona rodeada de cultivos manejados convencionalmente. Sin embargo, no están agresivamente intervenidos, ya que de acuerdo a Vilches (comunicación personal) no serían muchos los productos químicos utilizados en las labores agrícolas.

Este grupo está integrado por dueñas de casa que poseen en su mayoría huertas familiares, las cuales han manifestado su interés por diversificar la producción y mejorar el manejo que se hace de ellos. El terreno utilizado para la experiencia fue de manejo comunitario (en propiedad privada), donde se establecieron camas altas incorporando guano envejecido de oveja y tierra de hojas para mejorar la fertilidad, estructura, profundidad y drenaje del terreno. El predio contaba con obras menores de riego, a la que se le incorporó un sistema de riego por cintas presurizado por medio de un motor. Los cultivos establecidos fueron de hortalizas bajo un manejo orgánico los que se ubicaron en platabandas y con una disposición intercalada para impedir el desarrollo poblacional de cualquier plaga que pudiese estar en el huerto con anterioridad.

Un segundo grupo, Santa Rosa, se encuentra ubicado en la misma localidad de Chépica El terreno en que se realizó la experiencia se encuentra rodeado de cultivos manejados convencionalmente que en este caso, de acuerdo a Vilches (comunicación personal), incide negativamente en la producción orgánica establecida por el grupo. Este grupo está conformado por mujeres que están asociadas al grupo El Rincón, constituyendo la Sociedad R\&S. En este grupo e1 establecimiento de las flores se realizó en camas altas donde se plantaron diferentes variedades de gladiolos (Gladiolus hortelanus Bailey), además de claveles (Dianthus caryophilus L.), hortalizas y plantas medicinales. El manejo de Brevipalpus chilensis (arañita roja) se realizó mediante la aplicación de una solución de ajo y cebolla disuelta en agua aplicada con bomba de espalda y la fertilidad del suelo, mediante el uso de compostaje de los restos de la cosecha anterior, eliminando las plantas enfermas.

El tercer grupo, Rinconada de Meneses, se encuentra ubicado a $17 \mathrm{~km}$. de Chépica. Los terrenos con que cuenta este grupo se encuentran localizados al pie del cerro y posee cortinas cortavientos naturales, beneficiando la producción orgánica al no verse afectado por los cultivos colindantes. Este grupo lo componen mujeres campesinas, las que trabajan como temporeras en el periodo estival. Este grupo preparó camas altas a las que se le incorporaron guano y tierra de hojas. En ellas se establecieron zanahorias (Daucus carota) var. Chantenay; espinacas (Spinaca oleracea) var. viroflay; habas (Vicia faba L.) var. Morada; lechuga (Lactuca sativa L.) var. Rosita 4 estaciones; arvejas (Pisum sativum L.) y cebolla (Allium cepa). Para el manejo de pulgones (Aphis fabae) se 
realizaron aplicaciones de un preparado a base de ortiga (Urtica urens).

La postura epistemológica de este trabajo, por su naturaleza agroecológica, obedece a una perspectiva coevolucionista entre el sistema ambiental y el sistema social (Norgaard y Sikor, 1999), donde éste último está constituido por un sistema de conocimiento, valores tecnológicos y organizacionales, que están relacionados entre sí, y donde cada uno ejerce una presión selectiva en la evolución de los demás.

El nivel de indagación abarcó las tres perspectivas señaladas por Sevilla (2001b), distributiva, estructural y dialéctica, debido a que el manejo de los recursos naturales de los agroecosistemas estudiados fue abordado desde una perspectiva ecológica, socioeconómica y política, constituyendo niveles acumulativos de análisis.

El nivel de análisis utilizado fue el de agroecosistema, tanto en su dimensión ecológica como socioeconómica y desde un enfoque sistémico y que puede ser definido como un complejo sistema agrosocio-económico-ecológico (Conway, 1986) dentro de pequeñas unidades geográficas (Altieri, 1999a), producto de la alteración y manipulación, por parte del ser humano, de ecosistemas naturales, con el objetivo de establecer unidades productivas de carácter agrícola (Gliessman, 1998). Estos sistemas se caracterizan por ser abiertos, recibiendo insumos del exterior, dando como resultado productos que pueden ingresar en sistemas externos, y que contienen componentes abióticos y bióticos que son interdependientes e interactivos entre ellos (Altieri, 1999a) y con la organización social del ser humano (Minc y Vandermeer, 1990) y sus correspondientes atributos culturales (Mariaca, 1995) y tecnológicos (Ruiz, 1995).
Finalmente, en cuanto a las técnicas utilizadas (en lo concerniente a esta etapa de la investigación reseñada en este documento), hemos privilegiado las de carácter participativa y cualitativas o estructurales (Ortí, 1992). Estas corresponden a la observación participante y la entrevista semiestructurada (Tillman y Salas, 1994), o entrevista abierta semidirecta (Ortí, op.cit.).

Cabe hacer mención que por la naturaleza de la investigación (IAP), gran parte del marco teórico ha sido construido conforme se desarrolló el trabajo y en base a los antecedentes e informaciones obtenidas. Por razones de espacio no se encontrará ampliamente desarrollado como en su versión extensa.

\section{Resultados y Discusión}

La experiencia llevada a cabo por cada uno de estos grupos refleja situaciones particulares, que aunque podrían tener el mismo origen como lo es la implementación de tecnologías más sustentables, las dificultades que ello origina tiene causas diversas.

De esta manera si agrupamos de manera resumida y esquemática, las dificultades que los grupos presentaron a lo largo de esta experiencia, en problemas agronómicos, sociales y económicos, es posible señalar que para el caso del primer grupo, éste no presentó ningún problema de tipo agronómico, ya que la principal dificultad relativa a la sanidad de los cultivos, fue resuelta de manera eficiente, por lo que la producción no se vió mermada ni en cantidad ni calidad. Sin embargo, desde el punto de vista social, el principal problema lo constituyó el mantener cohesionado el grupo que se había conformado con estos propósitos. Precisamente, la principal razón de ello obedece a un problema de tipo económico, ya que no se establecieron eficientes canales de 
comercialización que le permitieran vender el volumen producido, a pesar de haber obtenido precios mayores que los de la feria local de Chépica. Para el caso del segundo grupo, la situación no difiere mucho, ya que también tuvieron problemas de organización, por una suerte de incompatibilidad entre los integrantes de ambos, ya que, al parecer, la asociación conformada, persigue fines más bien instrumentales de acceso a fuentes de financiamiento. A esto se suma el hecho que el manejo de los cultivos no se realizó de manera adecuada, sobre todo en lo que al manejo de plagas se refiere, lo que derivó en la obtención de una producción de mala calidad, y por consiguiente, la venta a precios inferiores al promedio transado en la feria. Finalmente, el tercer grupo supo enfrentar bien el manejo de los cultivos y lo que es más importante, consolidar el grupo, a pesar que éste se viera disminuido en el número de integrantes conforme se desarrollaba la experiencia. Este aspecto constituyó la principal fortaleza de este grupo donde los lazos familiares resultaron muy determinantes a la hora de salvaguardar el grupo.

Estas dificultades observadas del análisis de estas experiencias, nos permite establecer una serie de condiciones para conseguir, al menos, dar algunos pasos en este proceso de transición hacia sistemas agrarios más sustentables.

Los elementos o condiciones que hemos determinado para realizar una transición agroecológica a partir del establecimiento de unidades productivas de naturaleza orgánica y sobre la base de nuestra experiencia, son las que se detallan a continuación.

\section{Iniciativas Locales}

Con este primer elemento nos estamos refiriendo a que cualquier proceso que se desee implementar, transitar o recorrer (desarrollar en último término) debe, casi imperiosamente, surgir como una iniciativa de los propios campesinos involucrados, ya que serán ellos los que permanentemente demandarán y velarán por el cumplimiento de los objetivos trazados, además de imponer el ritmo de consecución de los mismos. De esta manera existirá un mayor compromiso por parte de ellos, al ser gestores de su propio proceso de desarrollo. Esto quedó demostrado en el presente estudio, ya que de los tres grupos iniciales con los que se trabajó, sólo uno de ellos demostró un real interés y motivación para seguir en este proceso, que a la postre fue con el que terminamos el trabajo. Cobra real importancia el hecho que la iniciativa surja desde los propios campesinos, ya que de esa manera se generan con mayor naturalidad y espontaneidad relaciones de igualdad e intercambio necesarias en un proceso agroecológico, y con ello niveles de involucramiento cada vez más crecientes, traduciéndose en mayores grados de participación y protagonismo durante el proceso.

Expresiones como "lo que a mí me gustaría es poder plantar algunos paltos", refleja esa inquietud de querer ser protagonista de la planificación de su propio predio, sustentando lo anteriormente expuesto.

\section{Programa de Acompañamiento}

Entendemos como programa de acompañamiento a aquel que se planifica para una determinada zona donde estarán incluidos aquellos grupos de campesinos abarcando la totalidad o no de las comunidades. El análisis de nuestra experiencia nos señala que estos programas deben a lo menos cumplir con las siguientes características:

a) Que responda a las necesidades sentidas por los campesinos, considerando la capacidad creativa de 
éstos, como respuesta a esta inquietud manifestada desde las bases mencionadas anteriormente. Entendemos necesidad como la carencia y la potencia (Max-Neef, 1996) de aquello que está oculto en un grupo de sujetos y que no habiendo sido develado para los ojos y la conciencia de los técnicos, puede manifestarse por medio de una acción colectiva.

b) Una segunda característica es que los programas de acompañamiento estén inmersos en un contexto socioeconómico, ya que en la mayoría de los casos los programas de desarrollo son concebidos sólo como un proyecto productivo, desatendiendo las otras funciones que la agricultura (actividad agraria) desempeña, como la autosuficiencia alimentaria, "mejorar la autoestima", la reproducción social y biológica de un agroecosistema, entre otras.

Importante es destacar que, en muchos de los casos, los programas de desarrollo no consideran el punto de vista del propio campesino respecto de su concepción del desarrollo en cuanto no sólo a los objetivos que se quiere alcanzar, sino también en los tiempos destinados para realizarlos, los que generalmente se condicen con escalas ecológicas de manejo.

A través de expresiones como " es dificil atender bien el trabajo, por que además de esto tenemos otras cosas que hacer", " es que nos vienen a enseñar cuando tenemos que ir a trabajar a la fruta y ahí pagan bien", nos podemos dar cuenta que no ha sido comprendida la lógica utilizada por los campesinos en su distribución de las actividades diarias.

Considerar las dimensiones tiempo y espacio implica captar la hermenéutica del investigado, rompiendo esquemas de jerarquización, para establecer relaciones simétricas que permitan una comunicación dialógica entre dos culturas que tienen que interactuar e interpretarse mutuamente.

c) Una tercera característica que, a nuestro juicio, deben cumplir los programas de acompañamiento, es que éstos deben contar con profesionales capacitados no sólo para el apoyo o acompañamiento en cuanto a técnicas que no sean degradantes con los recursos naturales, sino que tampoco lo sean con las relaciones sociales que se dan en la comunidad. El profesional debe además, poseer conocimientos en herramientas participativas que permitan ir generando un cúmulo de conocimientos (en conjunto con el campesino) durante el desarrollo del proceso agroecológico. La carencia de estas condiciones pudimos constatarlas en este estudio, ya que la asistencia -por que así fue concebida- de los técnicos, no fue capaz de resolver, no sólo problemas de tipo agronómico en algunos casos, como por ejemplo el control de pulgones, el manejo de los residuos de cosecha, la elaboración del compostaje-, sino que además fue insuficiente e insatisfactoria en términos de la planificación predial, siquiera para la programación de las cosechas con salida al mercado, a pesar que la orientación de ellas iba dirigida con esos fines. Ello quedó demostrado por el fracaso de unos de los grupos que habiendo obtenido una producción de hortalizas orgánicas dentro de parámetros de rentabilidad aceptables, no fue posible venderla en mercados locales. Otra de las carencias que pudimos observar en nuestro estudio fue que no se trabajó con la necesaria consistencia, por parte de los técnicos en lo relativo a la consolidación y afiatamiento de las organizaciones conformadas, lo que se reflejó en que en la totalidad de ellos, por ejemplo, el número de miembros se reducía conforme avanzaba la temporada, impidiendo la efectiva participación de sus integrantes y el 
intercambio de saberes como elemento potenciador y sinergético. Por otra parte, el discurso de los campesinos a través de expresiones como " no, no sacamos ná con preguntarles (a los técnicos), por que casi nunca tienen respuestas, nos dicen que lo van a ver y después nos van a decir" y "es que yo no entiendo las cosas que hay que hacer cuando nos explican", refleja un desconocimiento en materias de agricultura orgánica y la ausencia de metodologías participativas y de educación popular.

Sin embargo, el análisis de este argumento resulta más complicado de lo que parece, ya que no sólo se resuelve con la incorporación de profesionales mejores capacitados en estos conocimientos, sino que además se requiere la adoptación de una serie de actitudes (Salas, 1998) que posibilitan la implementación de técnicas participativas que son inherentes a la persona que investiga.

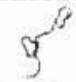

La formación de profesionales en materias de carácter agroecológicas, en lo relativo no sólo a to técnico, sino en aspectos sociales, económicos y culturales resulta indispensable para abordar procesos de transición agroecológica. Este problema en el ámbito formativo y de capacitación del profesional que se desempeña en el área del desarrollo rural, ha sido planteado por autores cuyas aportaciones teóricas han sido clasificadas dentro del pensamiento alternativo (Altieri, 1991; Sevilla y Woodgate, 1997). Organismos oficiales internacionales como la FAO (1988) en su informe sobre formación y capacitación de profesionales de ciencias agrícolas, tambien deja ver, aunque tímidamente, la necesidad de incluir, en lo formativo, disciplinas relativas a las ciencias sociales . Por otro lado, el reconocimiento que los resultados de la investigación y paquetes tecnológicos que se generan en las universidades y centros de investigación, no cubren la diversidad de situaciones socioeconómicas, tecnológicas y culturales, y tampoco se adecuan a la diversificación propia de los sistemas productivos de los pequeños agricultores.

$\mathrm{Al}$ respecto, nos hemos referido en otras ocasiones, a lo complejo que resulta la incorporación de enfoques agroecológicos en las mallas curriculares de carreras universitarias como la de Agronomía (Peredo, 1999 a y 2000 a), tema que no lo abordaremos en esta oportunidad ya que escapa a los objetivos de este trabajo.

d) Una cuarta y última característica que deben reunir los programas de acompañamiento es que éstos permitan la integración del grupo de campesinos con la sociedad mayor, para que de esa manera se articulen mecanismos de "interpretación" de las burocracias estatales, facilitando así, la autogestión de los grupos campesinos. Es aquí donde el profesional que trabaja con comunidades o grupos campesinos cumple un rol trascendental, ya que es éste quién realiza la tarea que definimos como de "interpretación" por ser quién más debería conocer la realidad global del mundo agrario. Además, en esta perspectiva, puede cumplir la tarea de ser el interlocutor entre la gente y la burocracia institucional. Para nuestro caso, pudimos comprobar que, efectivamente, no eran quienes entregaban la asistencia técnica del programa productivo quienes realizaban esa interpretación, sino "agentes" externos al programa, los que sí conocían a los campesinos y campesinas, su entorno y como ellos y ellas lo trabajaban. Es más, eran estos últimos los que en un proceso de acompañamiento, permitían salvar los obstáculos que se presentaban, que en la mayoría de los casos no eran de carácter técnico. 


\section{3.- Movilización de los Sujetos del Cambio}

Un tercer elemento que pudimos constatar que era fundamental en un proceso agroecológico, era el apoyo que se brindara a los grupos campesinos con lo que se trabajaba, apoyo que debería ser capaz de movilizarlos con el objetivo de organizarlos ya sea de manera formal o informal. De esta manera, podría ser posible canalizar las propuestas de cambio planteadas por los propios campesinos desde el punto de vista más instrumental, optando a servicios de asistencias de cualquier procedencia. Sin embargo, en nuestra investigación, pudimos comprobar que el proyecto llevado a cabo con los campesinos, no logró movilizarlos para constituir una organización formal, ya que en el proceso de constitución de las mismas, se desatendieron aspectos tan importantes como la confianza entre sus integrantes y los objetivos para los cuales se habían organizados. Lo anterior 10 observamos a partir de expresiones como " lo que pasó es que él se incorporó por que necesitábamos a alguien más y así nos daban la ayuda, pero después nunca trabajó", "yo trabajo mejor con mi prima y mi cuñada, por que les tengo confianza", "que sacamos con organizarnos si no recibimos más plata con lo que producimos".

La movilización de los campesinos permite además recuperar la confianza en sus propias capacidades y de esa manera tomar sus propias decisiones y poder negociarlas con las instituciones. Sin embargo, esta retórica se vuelve acción siempre que a estas alturas del proceso se haya logrado desplegar las potencialidades con que cuenta el grupo con el cual se está interactuando. Esto pudimos obscrvarlo claramente en nuestro caso, ya que con el grupo que seguimos trabajando, en las etapas posteriores se presentaron estas circunstancias señaladas anteriormente. Fue, precisamente, con este grupo donde además de la permanente inquietud manifestada por sus integrantes por implementar acciones concretas -las que incluso llevaron a retrasar algunas de las etapas previamente planificadasexistió, por parte de estos agentes externos al programa, la capacidad y la voluntad de acompañarlos en el proceso combinando dos aspectos centrales: por un lado la permanente actitud de incorporar aquellas iniciativas (de tipo técnico en lo referido al manejo del sistema productivo establecido) surgidas por las campesinas, o sea el conocimiento local; y por otro lado, la constante preocupación de informar y enseñar de las gestiones a realizar ante las distintas instituciones, o sea la interpretación.

\section{4.- Elección de la Propuesta Técnica.}

La elección de la propuesta técnica resulta determinante para sustentar, desde el punto de vista productivo, un proceso agroecológico. Constituye, además, un factor de evaluación cuantitativa y una posible causa del éxito o fracaso de un proyecto. Sin embargo, no solo debe responder a un objetivo meramente productivo, sino que además, por tener una naturaleza agroecológica, la propuesta técnica a implementar debe ser aquella que al menos considere el uso de recursos locales, que respete los ciclos biológicos y la reproducción de éstos y que no genere más residuos de los que el propio agroecosistema pueda reciciar.

Por tanto, la elección de una propuesta técnica adecuada reviste gran importancia, debido principalmente a tres aspectos: a) por el carácter agrario tanto de la economía como las relaciones sociales del campesino, b) por que debe obedecer a la naturaleza agroecológica en tanto proceso completo $y$, c) por el peso que ađquiere la dimensión productiva en los proyectos de desarrollo rural. 
a) Cuando nos referimos al carácter agrario de la economía campesina, nos estamos refiriendo a que toda propuesta agroecológica debe poner como centro y motor de la economía campesina a la producción agrícola, entendiéndola a ésta como un estilo de manejo de los recursos naturales del predio (Van der Ploeg, 1993) dentro de un contexto de mutifuncionalidad (Altieri, 1999b) y porque constituye el corpus y la praxis de la racionalidad ecológica del campesino (Toledo, 1993).

Lo anterior, por tanto, significa que la propuesta de acompañamiento agroecológica no debiera articularse sobre la base de actividades ajenas a la actividad agraria como una alternativa para el desarrollo rural. Diversos autores considerados clásicos de los estudios campesinos (Sevilla y Pérez, 1978), tanto antropólogos, sociólogos como economistas, ya sea de una visión ortodoxa como heterodoxa, pertenecientes a la antigua o nueva tradición (Palerm,1980) y bajo diferentes niveles de análisis (unidad familiar, comunidad campesina y cultura y sociedad), caracterizan al campesino, con o sin tierra, en su relación con la actividad agraria, destacando con ello el papel fundamental de la explotación agraria (Sorokin y Zimmerman, 1929; Kroeber, 1948; Steward, 1950; Redfield, 1956; Wolf, 1971; Shanin, 1976; Sevilla, 1976; Galeski, 1977; Sevilla y Pérez, 1978; Shanin, 1979; Sevilla, 1985; Toledo, 1993), y que por razones de espacio no nos detendremos en este aspecto (Peredo, 2001).

Con ello queremos hacer hincapié en la importancia de la explotación agraria o manejo de los recursos prediales en tanto unidad económica y social, donde se generan, por un lado, los comportamientos sociales, patrones de conducta, formas de interacción y valores que determinan la vida social de los individuos $y$, por otro, las actividades agrarias con la distribución de papeles del equipo de producción que constituyen la empresa (Sevilla,1985), confirmando la importancia que ésta tiene a la hora de proponer estrategias de desarrollo con base agroecológica.

Sin embargo, el otorgarle tal dimensión en lo económico, reconociendo que la economía campesina es, en último análisis, una forma particular de producción rural o agraria donde los productores utilizan los recursos naturaies como medios básicos e irremplazables (Toledo, 1993), no significa que desconozcamos la presencia de otras actividades productivas (Sevilla y Pérez, 1978 y Galescki, 1977), además de las no productivas que aportan al ingreso familiar. De esta manera no caemos en un enfoque agriculturista, sino que le damos crédito al enfoque de las estrategias del hogar (Rivera, 1988), como un enfoque multifuncional en la generación del ingreso familiar, pero que no cuestiona la definición del campesino como un pequeño productor agropecuario en condiciones de subordinación social.

b) El segundo aspecto que hemos considerado que reviste gran importancia a la hora de elegir una propuesta técnica es la naturaleza agroecológica del proceso global. Con ello nos estamos refiriendo que la tecnología a la que se recurra debe en lo ecológico, conservar la base de los recursos existentes, mediante técnicas como el cultivo múltiple, agroforestería, control biológico, reciclaje de residuos, entre otras (Simón, 1996), ya que la falta de sustentabilidad ecológica en el agroecosistema puede proceder no solo de la destrucción de los recursos renovables, sino que además, ser consecuencia de la utilización de tecnologías inadecuadas o de su inexistencia, conllevando a la pérdida de beneficios potenciales de los propios recursos internos del agroecosistema. En lo social la sustentabilidad implica, además, que la utilización de tecnologías 
procuren lo que Conway (1986) llama la equidad social y autonomía de los agroecosistemas.

Desde una dimensión social entendemos por equidad a la propiedad de los agroecosistemas de distribuir ecuánimemente la producción entre sus beneficiarios. Propiedad que no sólo está referida a la distribución del producto, sino que además de los beneficios netos (Conway y Barbier, 1990). Esta se logra cuando un sistema productivo puede hacer frente a crecimientos razonables de la demanda de alimentos sin que se incremente el costo social de producción. La equidad no sólo debe ser analizada en relación con la distribución de los productos agrícolas (y sus costos), sino que además con relación al acceso a los inputs. Característica que adquiere mucha relevancia cuando se determina una estrategia para implementar una agricultura de tipo orgánica dentro de un proceso agroecológico (Peredo, 1999c)

Desde una dimensión ecológica la autonomía del agroecosistema tiene que ver con el grado de integración o control de éstos. Esta propiedad se ve reflejada en el movimiento de materiales, energía e información entre sus partes componentes, y entre el agroecosistema y el ambiente externo (Altieri, 1999; Gliessmann, 1998).

Finalmente, desde una dimensión cultural, queremos señalar el activo papel que debe jugar el conocimiento local en el diseño de tecnologías y estrategias de producción y sobre las redes y agentes de socialización que a través de mecanismos de transmisión oral han compartido ese conocimiento autóctono a través de generaciones, permitiendo conservar la base de los recursos locales (Peredo y Fuentes, 2000b ). En nuestro caso, expresiones como "por ejemplo, eso que hacen con eso (relativo a un preparado), a nosotros nos resulta mejor con esto”, “ lo que pasa es que hay que colocar más plantas que le ayuden al suelo a darle fuerza, ese es el problema de éste (suelo)", " no, yo lo hago (abonera) de esta otra manera, por que sale mejor y nada más rápido"; reflejan la propia praxis de los campesinos que ha ido generando un conocimiento acerca de su entorno sobre la base de la experiencia acumulada. Sin embargo, hemos podido comprobar que ese conocimiento al no ser valorado puede perderse en las generaciones más jóvenes, como puede deducirse de esta expresión: “a veces, cuando mi papá nos ayuda resulta mejor, porque él sabe trabajar la tierra mejor que nosotros"

La incorporación de estas tres dimensiones (ecológico, social y cultural) a la hora de implementar cualquier tecnología en un proceso agroecológico, debe hacerse de manera holística, considerando las interrelaciones entre ellas. En nuestro caso, la implementación de técnicas generadas por los propios campesinos, ya sea de prácticas culturales ancestrales y/o de aquellas derivadas de la modificación y adaptación de la generada por la ciencia alternativa, ha permitido de manera gradual, el control parcial de algunos de los sistemas productivos, basándose en una gestión agroecológica de los recursos intraprediales a través de acciones colectivas por parte de los campesinos (Peredo et al, 2000).

En nuestro caso, pudimos constatar que no siempre se generan impactos positivos con la implementación de una agricultura de tipo orgánica (biológica o ecológica de acuerdo a Sevilla, 2000). Incluso se pueden originar impactos negativos en agroecosistemas calificados de "privilegiados" por ubicarse en zonas geográficamente aisladas y con una mínima interacción con predios manejados convencionalmente. El hecho que la implementación de una agricultura orgánica no genere siempre 
impactos positivos se debe, a nuestro parecer y bajo nuestras condiciones, a las siguientes situaciones observadas en nuestro estudio:

1.- Por la naturaleza de los programas de apoyo a la agricultura campesina, caracterizados por su heterogeneidad, en cuanto a que sus enfoques son parcelarios e inestabilidad (Gómez y Echeñique, 1989). Estos, cuando son de carácter productivo, se implementan sobre la base de sistemas de producción independientes que pocas veces se integran al agroecosistema completo. En la práctica se traduce en predios mosaicos donde intentan convivir sistemas productivos con distintos manejos, pero que no son interdependientes a la hora de realizar el manejo predial.

2.- Otra situación muy recurrente es que debido a las características y condiciones de los programas de asistencia técnica, se presionan los sistemas productivos orgánicos para que respondan positivamente al cabo de la primera temporada, desconociendo que la transición a manejos orgánicos se estabiliza en al menos de tres a cinco años (Gliessman et al, 1996).

3.- Una tercera situación que pudimos observar es que la implementación de sistemas productivos orgánicos en zonas precisamente "privilegiadas", como en nuestro caso, conlleva a un desequilibrio del sistema más que una tendencia a la estabilización ecológica, ya que por la condición de aislamiento y marginalidad (ecológica, socioeconómica y política), el tipo de manejo que se lleva realizando en estos lugares obedece a to que Alonso et al (2001) han definido como ecoagriculturas, que muchas veces resulta mucho más ecológica que la propia agricultura orgánica. Entonces, la introducción de un manejo de tipo ecológico provoca un cambio en la lógica de producción de los campesinos y una pérdida del control sobre su propio ecosistema, sobre todo cuando este tipo de agricultura también es dependiente de insumos externos. Estas situaciones obligan a replantearse las etapas establecidas en la transición agroecológica (a nivel productivo) entendiéndola como el proceso de cambio en las prácticas agrícolas en un proceso de desintensificación con una paulatina reducción de inputs de naturaleza industrial.

Si la propuesta agroecológica está orientada a la agricultura campesina (local e indígena), por que en ésta se sustentan sus principios, lo que corresponde al momento de elaborar propuestas de desarrollo basados en el manejo ecológico de los recursos naturales locales a través de una agricultura sostenible (Sevilla,1997) es establecer el nivel o grado de sustentabilidad del predio en que se actuará para definir, a partir del potencial específico de cada agroecosistema, la estrategia con que se implementarán los cambios planificados, ya que no siempre será necesario la aplicación rígida y consecutiva de las etapas pre-establecidas (en la teoría) para la transición agroecológica.

c) Retomando nuestra argumentación, señalamos por último el tercer aspecto de la importancia de la propuesta técnica de un proceso agroecológico, que dice relación con el carácter productivo que tienen los proyectos de asistencia. Ello obedece a que entre los objetivos planteados por estos últimos (Indap,1999), es posible observar que la dimensión productiva y la generación de excedentes ocupan un lugar muy importante, a la vez que sostienen la necesaria introducción de tecnologías agropecuarias que, además, logren un mejoramiento del medio ambiente. 
Sin embargo, no están explícitos cuáles deberían ser aquellas tecnologías con las cuales se podrían cumplir el doble objetivo de aumentar productividad y mejorar el medio ambiente. Esta omisión es la que ha permitido implementar "tecnologías amigables con el medio ambiente" y que finalmente, se han concretado en el diseño de sistemas orgánicos como los establecidos por los grupos de campesinos en cuestión.

De ahí la importancia de una adecuada implementación tecnológica teniendo presente que mediante esta vía administrativa (asistencia técnica estatal), que por el momento resulta una alternativa concreta y real para aquellos campesinos que practican una agricultura familiar, conlleva el cumplimiento de los objetivos anteriores en el brevísimo plazo de un año y cuya renovación está sujeta al logro de estos objetivos.

\section{5.- Resultados Productivos Exitosos}

Muy unido a la importancia de elegir una propuesta técnica adecuada, creemos necesario recalcar la importancia de obtener buenos resultados productivos en el más corto plazo posible, por estar sujetos a los objetivos trazados por los programas de asistencia técnica aludidos en el apartado anterior.

De ahí el hincapié que haga Yurjevic (1998) en su propuesta de desarrollo, donde contempla el carácter rentable y sustentable de los proyectos productivos de naturaleza agroecológica, haciendo alusión, precisamente, a la urgencia de los resultados positivos dentro del proceso de transición.

Por ello, adquiere mucha relevancia la estrategia que adoptan los campesinos para alcanzar los objetivos de producir excedentes para la venta sin degradar ni sobre explotar los recursos que manejan. Complejo es, por tanto, el desafío de cumplir con estos dos objetivos cuando de por medio está en juego la seguridad alimentaria de una familia. Es aquí, cuando una estrategia de agricultura orgánica resulta beneficiosa para estos fines. Sin embargo, su implementación tendrá un correcto sentido para la consecución de nuestros objetivos agroecológicos, si ésta se implementa dentro de una estrategia más amplia que implique el rediseño paralelo del predio, sin mayores inversiones en un principio y donde la producción orgánica se implemente de manera transitoria y sobre la base de los recursos (e insumos) que estén a su disposición en su entorno inmediato.

O sea, cuando señalamos la obtención de resultados productivos exitosos como un elemento fundamental para una estrategia agroecológica, lo hacemos por que estamos conscientes que es una condición exigida por las instituciones burocráticas y porque, es una manera de defender la replicabilidad de estos sistemas agroecológicos. Sin embargo, creemos necesario resaltar que ello se inserta en una estrategia agroecológica más compleja basada en la diversificación, donde la producción orgánica sería una etapa más y no el objetivo final.

El punto central, entonces, es la búsqueda de una estrategia que permita avanzar hacia estados superiores trazados a largo plazo que impliquen el desarrollo en plenitud de los potenciales ecológicos, humanos y sociales de un grupo de campesinos a través de etapas consecutivas, que estando condicionadas por las normativas impuestas por estas instituciones asistenciales, permitan un óptimo aprovechamiento de las ayudas otorgadas, y la renovación de los contratos establecidos (la casi totalidad de ellos anuales y sujetos a la consecución de objetivos productivos). Por tanto, la obtención de resultados positivos de aquellas unidades establecidas 
bajo apoyo técnico obedecería, más bien, a una táctica para retener las ayudas que brinda el aparataje estatal.

\section{6.- Presencia de Mercados Locales}

En los puntos analizados anteriormente, hemos señalado la importancia de la concertación del conocimiento y voluntad por parte de campesinos y técnicos para llevar a cabo de manera exitosa un proceso de transición agroecológica. Ahora, aparece un nuevo elemento, donde además es necesaria la concertación de otro actor social: los políticos, aquellos responsables de garantizar las condiciones que permitan la inserción y articulación de la agricultura familiar.

Si, como ya lo hemos discutido, una condición necesaria para recibir la ayuda por parte de los programas asistenciales, es que la producción genere un excedente, éste debe tener un mercado asegurado, ya que no se puede abandonar una iniciativa por carecer éste de un mercado comprador. De hecho, el análisis del proceso llevado a cabo por uno de los grupos de campesinos, deja en evidencia que el elemento que estuvo ausente, fue precisamente, el no contar con un mercado donde circulara el excedente generado por su producción hortícola, pese a haber obtenido una producción orgánica económicamente rentable y haber fortalecido socialmente al grupo, a través de la organización conformada. Esto significó la descontinuidad del programa asistencial con este grupo, con la consecuente desmotivación de los campesinos, que terminaron por disolver su organización al ver que ésta ya no cumplía con sus motivaciones. Ello queda de manifiesto en las expresiones " nos dijeron que las íbamos a vender mejor, pero al final no pasó ná...”, “ estaba todo bien, pero nadie nos compró las flores. Incluso una señora nos dijo que si las hubiésemos tenido para la fiesta de su hija al final del año, las hubiera comprado todas". Estas reflejan la ausencia de canales de comercialización y mercados locales para la venta de los excedentes que muchas veces están obligados a obtener, por los motivos discutidos en apartados anteriores.

A la luz de estos antecedentes y de la discusión que hemos venido realizando desde el enfoque agroecológico, pudiese resultar ambiguo y contradictorio el peso que se le quisiera dar a este punto, al situarlo como un aspecto relevante dentro de un proceso de transición agroecológica. Sin embargo, ello no es así, ya que lo que se coloca en discusión no es la presencia o ausencia de éste, sino el tipo de relaciones que establece el campesino con aquel.

Al señalar al mercado como un elemento gravitante dentro de un proceso agroecológico, en realidad a lo que estamos haciendo referencia es a una serie de elementos o condiciones que se deben reunir, a través de la concertación de los actores anteriormente señalados (campesinos, técnicos y políticos), traduciéndose en este elemento final. O sea, si consideramos a estos tres actores como negociadores de un proceso, a nuestro juicio se debería avanzar en conseguir que:

a) Los campesinos, mediante su lógica de organización social y de manejo de recursos naturales, consideren unidades o rubros productivos para la generación de excedentes.

b) Los técnicos, a través de su propuesta y asistencia de rubros orientados al mercado, incorporen criterios de planificación basados en una buena información, que permita la oferta de productos que efectivamente va a tener salida, previo a tener resuelto, la seguridad alimentaria del grupo familiar al que se apoya. 
c) Los políticos, entendida como la clase gobemante, deben crear las condiciones mínimas que permitan la circulación de bienes entre productores y consumidores, de manera justa y solidaria.

El apoyo y fomento de ferias locales para que se constituyan en lugares de mercadeo local basados en la confianza de las redes sociales establecidas en la región, puede constituir una actuación concreta que permita promover una relación de reproducción con carácter autónomo, entre los campesinos y el mercado, donde éste último se constituya en el destino final de la producción y no donde se movilicen los factores de la producción (Van der Ploeg, 1993).

Finalmente, creemos que se debiera apostar por una estrategia que permitiera el acceso oportuno a un mercado de carácter local, potenciando los circuitos cortos, y apostando por una estrategia de producción orgánica (más bien natural y/o tradicional) basada en la reducción de los costos como resultado del uso de insumos locales (o la progresiva independencia de insumos externos).Elio, respecto de la búsqueda del sobre precio (precio premio), debido a las características del mercado y la producción agroalimentaria chilena (Peredo, 1999b) que en última instancia incide en la economía familiar campesina, que es la que nos preocupa.

\section{7.- El Empoderamiento de los Campesinos}

Por último, planteamos que uno de los elementos claves para establecer una estrategia de transición agroecológica, pasa por que se cree un poder que radique en manos de los propios campesinos involucrados en procesos agroecológicos.

La generación de cualquier tipo de conocimiento, ya sea teórico y/o práctico, metodológico y/o tecnológico, debe ser asimilado y apropiado por parte de los campesinos con los cuales se está trabajando y acompañando, más aún cuando este conocimiento ha sido generado desce la cultura local producto de la interacción y diálogos de saberes con los técnicos.

Sobre la base de expresiones como "si el problema es que hay algunas cosas que se nos olvidan y no hallamos a quien preguntarle", "ya que no pueden venir siempre, podrían dejarnos algo...", y " yo creo que habria que poner más cosas. Si estas se dieron bien, yo me largo con más este otro año..." ; sumado a nuestras observaciones, hemos detectado al menos cuatro aspectos en los cuales corresponde actuar para el empoderamiento de los campesinos. Ellos son:

a) Apropiación de tecnologías: toda tecnología y/o práctica agrícola que se implemente para el manejo de los recursos naturales del agroecosistema, ya sean externos o generados localmente, debe ser incorporada por el campesino en un proceso constante de educación, con el fin de otorgarie autonomía y el control sobre los procesos productivos. De esta manera, la adopción de nuevas tecnologías debe entenderse como un proceso de asimilación a partir de experiencias locales (Peredo, 2000b)

b) Construcción de herramientas adecuadas que obedezcan a la lógica campesina, y que además permitan la interpretación de un lenguaje técnico muchas veces no comprendido por los primeros. De esta manera, no se genera una herramienta, que pese a haber sido generada sobre la base del conocimiento tradicional y local, en muchas ocasiones termina siendo una interpretación unidireccional de situaciones agroecosistémicas, donde el técnico obtiene mayores (a veces absoluto) beneficios de la información obtenida. Se debe evitar, por tanto, la generación de 
herramientas metodológicas engorrosas y complejas de implementar y que solo consiguen acumular información, dificultando su interpretación y análisis (Peredo, 1997). En la actualidad, esto es común observarlo en tomo a los discursos de la sustentabilidad que se han desarrollado con el objetivo de operativizar tal concepto basándose en posturas tecnocráticas y antropocéntricas (Daly y Gayo, 1995), que terminan siendo, en el ámbito agrícola, más bien una compleja interpretación de lo que algunos campesinos han realizado históricamente: una artificialización de ecosistemas a través de un manejo ecológico y perdurable de sus recursos naturales.

Sin embargo, en el proceso de construcción del concepto de sustentabilidad desde la agroecología (Peredo y Barrera, 2001b), es necesario a nuestro juicio, una educación popular que les permita a los grupos campesinos realizar una interpretación de aquel, para enfrentar procesos de negociación a la hora de su operativización bajo distintas formas de proyectos y/o programa de desarrollo

c) Un tercer aspecto corresponde al acceso a una información clara y oportuna, que continúa siendo un obstáculo para el desarrollo de grupos campesinos al momento de vender el excedente de producción que las propias instituciones de apoyo, tanto técnico como financiero, les exigen. Por lo que hemos considerado este punto como un importante aspecto en el cual se debe generar una estrategia de empoderamiento para que esta información, (precios justos y mercados locales) sea manejada y administrada por los propios campesinos sin la intervención de intermediarios o terceros. d) Poder de decisión y negociación. Finalmente, lo que debe lograr este empoderamiento de los campesinos, es una capacidad negociadora a la hora de decidir los elementos centrales de un programa y dirigir los procesos en los cuales se encuentran inmersos. En este aspecto, la agroecología puede y debe jugar un papel central, ya que las metodologías utilizadas ( $y$ otras en elaboración) potencian la participación campesina en los proyectos que ésta acompaña. Participación que, además, debe ser entendida como una negociación entre los diferentes sujetos involucrados (internos y externos), donde es fundamental la constante y permanente apropiación de elementos tanto metodológicos como tecnológicos, que permitan generar un poder de decisión, determinación y control sobre los procesos que se lleven a cabo de manera autónoma, bajo su lógica y formas organizativas.

Los antecedentes recién expuestos y que hemos denominado como condiciones necesarias y sobre todo de carácter preliminar para avanzar en un proceso de transición, obedece a lo que Costabeber (1998) define como la vía de la ecologización de la agricultura (en sus distintas dimensiones), donde el agricultor toma la decisión individual por un estilo de agricultura ecológica. Al parecer, esta estrategia toma mayor relevancia que la de la acción colectiva, dada la actual situación de desarticulación de la agricultura familiar, lo que llevaría mucho tiempo en reportar cambios. Sin embargo, por supuesto que no se descarta esa posibilidad, ya que si hay algo que caracteriza a estos proceso de transición agroecológica es su naturaleza multidimensional, donde la vía de la acción colectiva tarde o temprano resulta necesaria dentro de una estrategia más compleja que permita la puesta en común de las experiencias. 
Se trata, entonces, de establecer una estrategia sobre la base de las condiciones particulares de un grupo de agricultores y a partir de una situación concreta de cobertura técnica propio de un extensionismo rural que debiera evolucionar hacia un extensionismo que se caracterizara por ser un proceso de continuo aprendizaje para construir un conocimiento sobre la base de principios agroecológicos fundamentales como los son el enfoque sistémico y holístico, que le permita al agricultor tener el control sobre los procesos productivos para llevar a cabo un proceso de transformación consciente a través de la potenciación de las formas tradicionales de hacer agricultura como una vía de ecologización de la misma (Caporal, 1998).

\section{Conclusiones}

Conforme con los resultados discutidos, podemos concluir que es posible llevar a cabo procesos de transición agroecológica a partir del establecimiento de unidades productivas de naturaleza orgánica. Sin embargo, ésta debe ir inserta dentro de un proceso de desarrollo endógeno más global en donde, al menos, se concerten las condiciones o elementos que le otorgan dinamismo al proceso y que hemos definido como iniciativas locales, programa de acompañamiento, movilización de los sujetos del cambio, elección de la propuesta técnica, resultados productivos exitosos, mercados locales y el empoderamiento de los campesinos.

$\mathrm{El}$ orden en que hemos entregados estos elementos obedece a una secuencia en donde cada uno de elios se encuentra interrelacionado entre sí comenzando con las iniciativas que puedan surgir al interior de un grupo de campesinos o una comunidad para culminar con el empoderamiento de ellos mismos luego de un proceso llevado a cabo.
La vinculación de estos elementos por medio de una lógica dialéctica (tipo espiral) le otorga flexibilidad y dinamismo a la secuencia planteada permitiendo la identificación de diferentes fases en las que un proceso de naturaleza agroecológica puede acompañar a otro que esté en marcha, así como, apoyar iniciativas para generar sinergia entre los distintos sujetos.

De esta manera, se articulan los elementos de carácter endógeno y externos para reforzar una identidad (o construirla donde se haya perdido) a partir del potencial tanto natural como humano y definir esquemas de desarrollo endógeno. 


\section{Bibliografía}

ALONSO A, E SEVILLA y G GUZMAN (2001). Propuestas alternativas a la agricultura industrializada: el caso de la agricultura ecológica en España. Actas VII Congreso Español de Sociologí. Salamanca, 20-22 de septiembre.

ALTIERI M A (1987). Agroecology. The scientific basis for alternative agricultural. Westvicw Press Boulder, C.O.

ALTIERI M A (1991). Incorporando la Agroccología al Currículo Agronómico. Texto base de la reunión CLADES / FAO sobrc Agroecología y Enseñanza Agrícola en las Universidades Latino Americanas. Santiago de Chile, 2-6 septiembrc.

ALTIERI M A (1995). El estado del arte de la agroccología y su contribución al desarrollo rural en América Latina. En: Agricultura y desarrollo sostenible. Alfredo Cadenas (ed). Serie de estudios. Ministerio de Agricultura, Pesca y Alimentación. (España) 151-204pp.

ALTIERI M A (1999)a. Agroecología: Bases Cienlíficas para una Agricultura Sustentable. Nordan-Comunidad. Montevideo-Uruguay. 338p.

ALTIERI M A (1999b). Dimensiones Multifuncionales de la Agricultura Ecológica en América Latina. PED-CLADES / CIED. Lima-Perú. 82p.

ALTIERI M A y C NICHOLLS (1999). Agroecología. Teoría y Práctica para una Agricultura Sustentable. Red de Formación Ambiental. PNUMA.

CAPORAL F R (1998). La Extensión Agraria del Sector Público ante los Desafíos del Desarrollo Sostenible: el caso de Río Grande do Sul, Brasil. Tesis Dr. Ing. Agr. Universidad de Córdoba. $517 \mathrm{p}$.

CONWAY G (1986). Agroecosystem Analysis for Research and Development. Winrock International. Bangkok. 111p.

CONWAY G y E BARBIER (1990). After the Green Revolution: Sustainable Agriculture for Dev-Elopment. Earthscan Publications, London.

COSTABeber J A (1998). Acción Colectiva y Procesos de Transición Agroecológica en Río Grande do Sul, Brasil. Tesis Dr. Ing. Agr. Universidad de Córdoba. 422p.
CHONCHOL J (1994). Sistemas Agrarios en América Latina. De la Etapa Prehispánica a la Modernización Conservadora. Fondo de cultura económica. 443 p.

DALY H Y D GAYO (1995).Significado, conceptualización y procedimientos operativos del desarrollo sostenible: posibilidades de aplicación a la agricultura. En: Agricultura y desarrollo sostenible. Alfredo Cadenas (ed). Serie de estudios. Ministerio de Agricultura, Pesca y Alimentación. (España) 19 $38 \mathrm{pp}$.

DIAZ M (1995). Chile: programas rurales del fondo de solidaridad e inversión social. Lecciones y experiencias en un escenario de apertura y competitividad cconómica. En: Memorias del Seminario-Taller Internacional: El desarrollo rural en América Latina hacia el siglo XXI, Tomo IIExpcriencias.19-36pp.

ORGANIZACIÓN MUNDIAL PARA LA ALIMENTACION Y LA AGRICULTURA (FAO) (1988). Informe de los Seminarios Subregionales sobre Formación y Capacitación dc Profesionales de Ciencias Agrícolas para La Extensión y E! Desarrollo Rural en América Latina y El Caribe. Organización de la Naciones Unidas para el Desarrollo .13p.

GALESKI B (1977). Sociología del Campesinado. Ediciones Península. Barcelona.

GLIESSMAN, S. 1998. Agroecology. Ecological Processes in Sustainable Agriculture. Ann Arbor Press. USA. 357pp.

GLIESSMAN S, M WERNER, S SWEZEY, E CASWELL, J COCHRAN y F ROSADO-MAY (1996). Conversion to Organic Strawberry Management Changes Ecology Processes. California Agriculture 50 (1): 24-31.

GOMEZ S y J ECHEÑIQUE (1989). La Agricultura Chilena. Las dos caras de la modernización. FLACSO-AGRARIA. Santiago de Chile. 304p.

GUHA R y M GADGIL (1993). Los hábitats en la historia de la humanidad. En M González de Molina y J Martinez Alier. (ed). Historia y Ecología. Marcial Pons. Madrid. 49-110 pp.

GUZMAN G, A ALONSO, Y POULIQUEN y E SEVILLA (1996). Las metodologías participativas de investigación: un aporte al desarrollo local cndógeno. En: Actas del II Congreso de la Sociedad Española de Agricultura Ecológica. PamplonaIruña, septiembre. 301-316pp. 
GUZMAN G, M GONZALEZ DE MOLINA Y E SEVILLAGUZMAN (2001).Introducción a la agroecología como desarrollo rural sostenible. Ediciones mundi-prensa, Madrid.

INSTITUTO DE DESARROLLO AGROPECUARIO (INDAP). 1999. Camino al 2000. Ministerio de Agricultura del Gobierno de Chile. http://www.indap.cl

KROEBER A L (1948). Antropology. Harcourt Braceco, Nueva York.

MAX-NEEF M (1996). Desarrollo a Escala Humana. Ecoteca. Uruguay.

MARIACA R (1995). Agroecosistema, concepto central en la agroecología: búsqueda del desarrollo de un modelo aplicativo. En: Agroecología y Desarrollo Sustentable. L. González, V. De la Cruz, J. Aguilar, V. González, D. Delgado y A. Vargas (eds.) Universidad Autónoma de Chapingo. México. 91-102pp.

MINC y J VANDERMEER (1990). Intercropping. En: Agroecology. Carroll, R; J. Vandermeer Y P.Rosset (eds.) Mac Graw-Hill Publishing Company. USA. 481-516pp.

NORGAARD R y T SIKOR (1999). Metodología y Práctica de la Agroecología. En Agroecología. Bases científicas para una agricultura sustentable. Altieri, M.A. Nordan-Comunidad Montevideo. 31-46pp.

OFICINA DE ESTUDIOS Y POLITICA AGRARIA (2001). Actas de acuerdo de la mesa para el desarrollo de la agricultura familiar campesina. http://www.odepa.gob.cl/ mesacampesina.html

ORTI A (1992). La Apertura y el Enfoque Cualitativo o Estructural: la entrevista abierta semidirectiva y la discusión đe grupo. En: El análisis de la realidad social. García, M (comp.). Alianza Universidad. Madrid.171-202pp.

PALERM A (1980). Antropología y Marxismo. Nueva Imagen. México.

PEREDO S F (1997). Evaluación de la Calidad de Vida de una Comunidad Rural Mapuche de la región de la Araucanía. Tesis Ing. Agr. Universidad Austral de Chile.

PEREDO S F (1999a). El enfoque agroecológico en los programas curriculares para incorporar la dimensión ambiental en la educación universitaria. En Actas del II Congreso de Educación Ambiental para el Desarrollo Sostenible, II
Convención Internacional sobre Medio Ambiente y Desarrollo. Palacio de las Convenciones, La Habana, Cuba. Junio 14-18.

PEREDO S F (1999b). Agroecología. Una alternativa para el Desarrollo Campesino. Ponencia presentada en el Encuentro Nacional de Profesionales del Servicio País, Comité Nacional para la Superación de la Pobreza. Picarquín, Chile Septiembre.

PEREDO S F (1999c). De los Estilos de Agricultura Ecológica a la Agricultura Orgánica como Herramienta para la Agroecología. En Actas de las V Jornadas Cientificas de Estudiantes de Agronomía de Chile. Universidad Austral de Chile. Septiembre 4-7.

PEREDO S F (2000a). El Desafío del Enfoque Agroecológico para una Nueva Propuesta Curricular en la Formación de Agrónomos: la urgencia de la innovación. En Actas de las XII Jornadas de Extensión Agrícola. Universidad Católica de Temuco, Chile. Octubre 25-27.

PEREDO S F (2000b). Condiciones y factores necesarios para la adopción de tecnologías en los procesos agroecológicos. Conferencia presentada en el III Encuentro de Ingenieros en Agroecología. Universidad Autónoma de Chapingo, México. Septiembre 28-29.

PEREDO S F (2001). La Construcción de Indicadores de Sustentabilidad: una Negociación entre Sujetos. Tesis de Maestría en Agroecología y Desarrollo Rural Sostenible. Universidad Internacional de Andalucía.

PEREDO S F y E SEVILLA (2000). El Origen de la Agroecología: fundamentos para su conceptualización. Conferencia presentada en el Seminario Agroecologia: elementos para la gestión de la agricultura sustentable y el desarrollo rural humano. DRC-Colchagua, CIAL, INDAP, MAELA. San Fernando, Chile. Mayo 31.

PEREDO S F y P FUENTES (2000). Usos y Socialización del Conocimiento Tradicional de los PFnM: el caso de Armerillo. En Actas del II Encuentro de Investigación y extensión de los PFnM del Bosque Chileno. WWF, Red PFnM-Chile, UCM, CODEFF, INTEC, junio. Valdivia

PEREDO S F, F LEON y V VILCHES (2000). Accionar de las Organizaciones Campesinas para el Mancjo Agroecológico de los Recursos Naturales a Nivel Predial. En Actas del V Congreso Internacional de gestión en recursos naturales. CEA. Valdivia, Chile. Noviembre 20-24. 
PEREDO S F y C BARRERA (2001 a). Biodi versidad cultural y ecológica: elementos para el desarrollo rural endógeno. II Feria de la Biodiversidad Agricola. Plataforma Rural, Centro de Estudios Rurales y Agricultura Internacional, Valencia, España. Scpticmbre 14-16.

PEREDO S F y C BARRERA (2001b). La Sostenibilidad Agraria: un Proceso de Negociación. En Actas [I Con「erencia Cicntífica Internacional, Medio Ambiente Siglo XXI. Universidad Central "Marta Abreu" de las Villas, Santa Clara, Cuba. Noviembre 20-23.

REDFIELD R (1956). Peasent Society and Culture. The University of Chicago Press. USA.

RIVERA R (1988). Campesinado: el enfoque de las estrategias del hogar. Estudios Rurales latinoamericanos 12(3):327-362.

RUIZ O (1995). Agroecosistema: el término, concepto y su definición bajo el enfoque agroccológico y sistémico. . En: Agroecología y Desarrollo Sustentable. L. González, V. De la Cruz, J. Aguilar, V. González, D. Delgado y A. Vargas (eds.) Universidad Autónoma de Chapingo. México. 103-114pp.

SALAS M (1998). Epistemología y Participación. Documento de trabajo de la IV Maestria en Agroecologia y Desarrollo Rural Sostcnible de la Universidad Internacional de Andalucía, Seđe Iberoamericana de la Rábiđa, Huelva-España.

SEVILLA E (1976). The Peasentry and the Francoist Regime. En: Spain in crisis. P. Preston (cd.) Brighton, Harvester.

SEVILLA E (1985). El Campesinado. En: Tratado de Sociología. S. del Campo (ed.) Taurus. Madrid. 366-399pp.

SEVILLA E (1997). La Agroecología como Marco Teórico para el Desarrollo Rural. En: Paisaje y desarrollo integral en áreas de montaña. VII Jornadas sobre el Paisaje. Ministerio del Medioambiente. Madrid. 135-150pp.

SEVILLA E (2000). Estilos de Agricultura Ecológica. En: Introducción a la agroecología como desarrollo rural sostenible. G.Guzmán, M.González de Molina y E. Scvilla (eds.). Mundi-Prensa. Madrid.61-80pp.

SEVILLA E (2001a). Agroecología y desarrollo rural sosteniblc:una propuesta desde Latinoamerica. En: Agroecologia y agricultura sustentable. S Sarandon (ed).Universidad de la Plata: La Plata. Argentina.
SEVILLA E (2001b). La Perspectiva Sociológica en la Agroccología. II Jornadas Internacionales sobre Agroecología. Porto Alegre, Brasil. Noviembre.

SEVILLA E y M PEREZ (1978). Para una Definición Sociológica del Campcsinado. Agricultura y Sociedad $\mathrm{n}^{\circ} 1$ : octubre-diciembre. 15-38pp.

SEVILLA E y M GONZALEZ de MOLINA (1993). Ecología, campesinado c historia. La Piqueta. Madrid.

SEVILLA E y G WOODGATE (1997). Sustainable Rural Development: from industrial agriculure to agroecology. En: The International Handbook of Enviromental Sociology. M.Redelift y G. Woodgatc (eds.). Cheltenham. UK. 83-100pp.

SHANIN T (1976). Definiendo al Campesinado: Conceptualizaciones y desconceptualizaciones. Agricultura y Sociedad 11. España.

SHANIN T (ed.). (1979). Campesinos y Sociedades Campesinas. Fondo de Cultura Económica. México.

SIMON X (1996). El Desarrollo Rural Sustentable: una perspectiva agroecológica. Material preparado para la II Maestría en agroecología y Desarrollo Rural Sostenible. Universidad Internacional de Andalucía, sede Ibcroamericana de la Rábida, Huelva-España. 10p.

SOROKIN P y C ZIMMERMAN (1929). Principles of Rural Urban Sociology. Holt, Nueva York.

STEWARD IH (1950). Area Research: Theory and Practice. Social Research Bulletin, $\mathrm{n}^{\circ} 63$.

TILLMANN H y M A SALAS (1994). Nuestro Congreso. Manual de Diagnóstico Rural Participativo para la Extensión Campesina. PRODAF-GTZ. Costa Rica. 180p.

TOLEDO V M (1993). La Racionalidad Ecológica de la Producción Campesina. En: Ecología, campesinado e Historia. E. Sevilla y M.González de Molina (eds.). La Piqueta. Madrid. 197-218pp.

TOLEDO V M (1994). La apropiación campesina de la naturaleza: un análisis etnológico. México.

TOLEDO V M (2000). La paz en Chiapas: ccología, luchas indigenas y modernidad alternativa.Ed. Quinto Sol-UNAM, México. 
TOLEDO V M (2001).Biocultural diversity and local power in México. En Maffi L (Ed). On biocultural diversity linking language, knowledge and the environment. Smithsonian Institution. Press 472-488pp.

VAN DER PLOEG J (1993). El proceso de trabajo agrícola y la mercantilización. En: Ecología, campesinado e historia. Sevilla y Glez de Molina (eds.) Madrid: La Piqueta. 153196 pp.

WOLF E (1971). Los Campesinos. Editorial Labor. Barcelona. 151p.

YURJEVIC A (1998). Enfoque y Estrategia del Desarrollo Rural Humano y Agroecológico. Agroecología y Desarrollo 13 Diciembre: 6-10. CLADES. 